\title{
A key metabolic integrator, coenzyme $A$, modulates the activity of peroxiredoxin 5 via covalent modification
}

\author{
Jovana Baković ${ }^{1}$ - Bess Yi Kun Yu ${ }^{1}$ - Daniel Silva ${ }^{1}$ - Sew Peak Chew ${ }^{2}$. Sangeun Kim ${ }^{3}$. Sun-Hee Ahn ${ }^{3}$. \\ Laura Palmer $^{1} \cdot$ Lujain Aloum $^{1}$. Giacomo Stanzani ${ }^{4,5}$. Oksana Malanchuk ${ }^{6} \cdot$ Michael R. Duchen $^{4} \cdot$ Mervyn Singer $^{5}$. \\ Valeriy Filonenko ${ }^{6} \cdot$ Tae-Hoon Lee $^{3} \cdot$ Mark Skehel $^{2} \cdot$ Ivan Gout $^{1}$ (I)
}

Received: 22 January 2019 / Accepted: 20 July 2019 / Published online: 2 August 2019

(c) The Author(s) 2019

\begin{abstract}
Peroxiredoxins (Prdxs) are antioxidant enzymes that catalyse the breakdown of peroxides and regulate redox activity in the cell. Peroxiredoxin $5(\operatorname{Prdx} 5)$ is a unique member of Prdxs, which displays a wider subcellular distribution and substrate specificity and exhibits a different catalytic mechanism when compared to other members of the family. Here, the role of a key metabolic integrator coenzyme A (CoA) in modulating the activity of Prdx 5 was investigated. We report for the first time a novel mode of Prdx 5 regulation mediated via covalent and reversible attachment of CoA (CoAlation) in cellular response to oxidative and metabolic stress. The site of CoAlation in endogenous Prdx 5 was mapped by mass spectrometry to peroxidatic cysteine 48. By employing an in vitro CoAlation assay, we showed that Prdx5 peroxidase activity is inhibited by covalent interaction with $\mathrm{CoA}$ in a dithiothreitol-sensitive manner. Collectively, these results reveal that human $\operatorname{Prdx} 5$ is a substrate for CoAlation in vitro and in vivo, and provide new insight into metabolic control of redox status in mammalian cells.
\end{abstract}

Keywords Peroxiredoxin $5(\operatorname{Prdx} 5) \cdot$ Coenzyme A $(\mathrm{CoA}) \cdot$ Oxidative stress $\cdot$ Redox regulation $\cdot$ Reactive oxygen species (ROS)

Jovana Baković and Bess Yi Kun Yu have equally contributed to this manuscript.

Ivan Gout

i.gout@ucl.ac.uk

1 Department of Structural and Molecular Biology, University College London, London WC1E 6BT, UK

2 Biological Mass Spectrometry \& Proteomics Cell Biology, MRC Laboratory of Molecular Biology, Cambridge CB2 0QH, UK

3 Department of Oral Biochemistry and Department of Molecular Medicine (BK21plus), Dental Science Research Institute, School of Dentistry, Chonnam National University, Gwangju, Republic of Korea

4 Department of Cell and Developmental Biology, University College London, London WC1E 6BT, UK

5 Bloomsbury Institute of Intensive Care Medicine, University College London, London WC1E 6BT, UK

6 Institute of Molecular Biology and Genetics, National Academy of Sciences of Ukraine, Kyiv 03680, Ukraine

\author{
Abbreviations \\ PRDX5 Peroxiredoxin 5 \\ SBP Streptavidin binding protein \\ LC-MS/MS Liquid chromatography tandem-mass \\ spectrometry
}

\section{Introduction}

Peroxiredoxins (Prdxs) are antioxidant enzymes that neutralise a broad range of reactive oxygen and nitrogen species, including $\mathrm{H}_{2} \mathrm{O}_{2}$, alkyl hydroperoxides and peroxynitrites. Prdxs are highly conserved throughout evolution and broadly distributed from bacteria to humans $[1,2]$. The family of mammalian Prdxs can be subdivided into three major subclasses based on sequence, structural homology and catalytic mechanism: typical 2-cysteine (2-Cys) Prdxs (Prdxs14), atypical 2-Cys Prdx (Prdx5) and 1-Cys Prdx (Prdx6). Prdxs exhibit different subcellular localisation, regulation and substrate specificity. All members of the Prdx family share a common mechanism of peroxide-reducing activity that involves oxidation of a conserved peroxidatic cysteine (CysP) to a sulphenic acid (R-SOH), but differ in the mode 
of its reduction back to a thiol. The activity and cellular functions of Prdxs are tightly controlled by regulatory interactions and post-translational modifications. A diverse range of binding partners for Prdxs have been identified, including proteins involved in signal transduction, metabolic processes and antioxidant defence [3-5]. Reversible thiol modifications, including S-sulphenylation, S-sulphinylation, S-glutathionylation and S-nitrosylation, as well as phosphorylation and acetylation were shown to play key roles in regulating peroxidase activity, subcellular localisation and redox signalling of Prdx family members [3-6]. By regulating intracellular peroxide levels, Prdxs were shown to participate in the regulation of cell proliferation, differentiation and redox signalling under physiological and pathological conditions $[1,7,8]$.

Prdx 5 is the most divergent isoform among mammalian peroxiredoxins and shows a surprisingly wide subcellular distribution, including the mitochondria, peroxisomes, nucleus and cytoplasm [6]. The catalytic mechanism of $\operatorname{Prdx} 5$ is initiated by the reduction of a peroxide substrate, during which the peroxidatic Cys48 thiol (Cys48-SH) is oxidised to sulphenic acid (Cys48-SOH). The resolving Cys152 then reacts with Cys48-SOH to form an intramolecular disulphide. Due to the large distance between Cys152$\mathrm{SH}$ and $\mathrm{Cys} 48-\mathrm{SOH}$, the enzyme undergoes a significant conformational change to allow the two groups to come into proximity. Thioredoxins ( $\operatorname{Trx} 1$ in the cytosol or $\operatorname{Trx} 2$ in the mitochondria) reduce the disulphide bond (Cys48SS-Cys152) and regenerate a sulfhydryl group on Cys48, thereby reactivating the enzyme. This reaction requires the reductant NADPH, which is used by thioredoxin reductase (TrxR) to regenerate Trxs.

Consistent with its antioxidant properties, the knockdown of Prdx5 makes cells more susceptible to apoptosis induced by oxidative stress, while its overexpression prevents programmed cell death [9]. Transcriptional or translational upregulation or downregulation of the Prdx 5 gene was shown to take place in response to various stresses and during pathophysiological conditions such as cancer [7].

Coenzyme A (CoA) is an obligatory cofactor in all living cells synthesised from pantothenate (Vitamin B5), adenosine triphosphate (ATP) and cysteine [10]. CoA and its thioester derivatives (acetyl CoA, malonyl CoA and succinyl CoA among many others) are key players in major catabolic and anabolic pathways and the regulation of gene expression [10-13]. Many human pathologies, including cancer, diabetes and neurodegeneration, have been associated with abnormal biosynthesis and homeostasis of CoA and its derivatives [14-16].

We have recently discovered a novel non-canonical function of $\mathrm{CoA}$ in redox regulation, involving covalent attachment of this coenzyme to cellular proteins in response to oxidative and metabolic stress, termed protein CoAlation.
The development of new research tools and methodologies has been instrumental in uncovering protein CoAlation as a widespread post-translational modification and revealing its role in redox regulation. These developments afford us the unique opportunity to readily detect protein CoAlation in eukaryotic and prokaryotic cells in response to oxidative and metabolic stress and identify CoAlated proteins. To date, nearly one thousand proteins have been found to be CoAlated in bacteria, mammalian cells and tissues in response to oxidising agents and metabolic stress $[17,18]$.

Here, we report for the first time that Prdx 5 is covalently modified by CoA when cells and tissues are exposed to oxidative and metabolic stress. Mass spectrometry analysis revealed that the peroxidatic Cys48 is CoAlated in cellular response to oxidative stress. We demonstrated by employing an in vitro CoAlation assay that covalent binding of CoA to Prdx 5 results in complete inhibition of its peroxidase activity, which is reversed by reduction with DTT. Based on these findings, we propose that covalent modification of peroxidatic cysteine 48 in Prdx 5 in response to oxidative and metabolic stress not only protects it from overoxidation but may function as regulatory mechanism of redox signalling.

\section{Materials and methods}

All common chemicals were obtained from Sigma-Aldrich unless otherwise stated. The generation and characterisation of the anti-CoA antibody (1F10) was described previously [19]. Anti-Prdx 5 rabbit antibody were obtained from Abcam and secondary Alexa Fluor 680 goat polyclonal anti-mouse IgG and Alexa Fluor 800 goat polyclonal anti-rabbit IgG were from Life Technologies.

\section{Cell culture}

HEK293/Pank1 $\beta$ cells were generated as previously described [17]. Cells were maintained in DMEM (Lonza) supplemented with $10 \%$ foetal bovine serum (FBS, Hyclone) and $10 \mathrm{ml} / \mathrm{L}$ Penicillin-streptomycin (P/S, Lonza).

\section{Cardiomyocytes preparation}

Cardiomyocytes were isolated from adult male Wistar rats weighing 300-350 g. Primary cardiomyocytes were isolated as previously described with minor changes [20]. The isolated cardiomyocytes were suspended in M199 media (ZenBio) supplemented with $5 \mathrm{mmol} / \mathrm{l}$ creatine, $5 \mathrm{mmol} / \mathrm{l}$ taurine, $2 \mathrm{mmol} / \mathrm{l}$ carnitine, $100 \mathrm{IU} / \mathrm{ml}$ penicillin and $100 \mathrm{IU} /$ $\mathrm{ml}$ streptomycin, then plated on $6 \mathrm{~cm}$ dishes (VWR UK) precoated with $5 \mathrm{mcg} / \mathrm{ml}$ laminin. Cells were plated at a density of 340.000 rod-shaped cells per dish and incubated overnight at $37{ }^{\circ} \mathrm{C}$ and $5 \% \mathrm{CO}_{2}$ before experiments the following 
day. $>90 \%$ of attached cells appeared to be rod-shaped and non-contracting.

\section{Plasmids and transient transfection}

Human Prdx 5 cDNA without the signal sequence was cloned into the pCR3.1/Prdx5 vector as previously described [21]. HEK293/Pank1 $\beta$ cells were transfected at $\sim 60 \%$ confluence with pCR3.1-MAR plasmid vectors encoding streptavidin binding protein (SBP); SBP-tagged full-length WT-hPrdx5, hPrdx5C48S, hPrdx5C152S and hPrdx5C48/152S. Cells were transfected according to the manufacturer's protocol using Turbofect reagent (Thermo Scientific).

\section{Oxidative and metabolic stress induction}

Following transient transfection or an overnight sub-culturing, cells were incubated for $18 \mathrm{~h}$ in pyruvate-free DMEM supplemented with $5 \mathrm{mM}$ glucose, $10 \%$ FBS and $10 \mathrm{ml} / \mathrm{L}$ $\mathrm{P} / \mathrm{S}$. Pyruvate was removed from the media because it can act as an antioxidant and inactivate ROS. Cells were then treated with or without $\mathrm{H}_{2} \mathrm{O}_{2}(500 \mu \mathrm{M})$, diamide $(500 \mu \mathrm{M})$ or menadione $(50 \mu \mathrm{M})$, and incubated for $30 \mathrm{~min}$ at $37^{\circ} \mathrm{C}$. To induce metabolic stress, the medium of transiently transfected cells was replaced with pyruvate- and glucose-free DMEM and cells were incubated for an additional $18 \mathrm{~h}$. To allow recovery, full DMEM media was re-introduced after $18 \mathrm{~h}$ and cells were incubated in at $37^{\circ} \mathrm{C}$ for $30 \mathrm{~min}$.

\section{Cell lysis, immunoprecipitation and western blot analysis}

Harvested cells were lysed for 20 min in lysis buffer (at $4{ }^{\circ} \mathrm{C}$ ) containing $50 \mathrm{mM}$ Tris- $\mathrm{HCl} \mathrm{pH} 7.5,150 \mathrm{mM} \mathrm{NaCl}, 5 \mathrm{mM}$ ethylenediaminetetraacetic acid (EDTA), $50 \mathrm{mM}$ sodium fluoride $(\mathrm{NaF}), 5 \mathrm{mM}$ tetra-sodium pyrophosphate $\left(\mathrm{Na}_{4} \mathrm{P}_{2} \mathrm{O}_{7}\right)$ and $1 \%$ Triton $\mathrm{X}-100$, supplemented with fresh $100 \mathrm{mM}$ NEM and fresh $1 \times$ Protease Inhibitor Cocktail (PIC, Roche). Total cell lysates were centrifuged at $20,817 \times g$ for $10 \mathrm{~min}$ at $4{ }^{\circ} \mathrm{C}$ and the supernatant was collected for further analysis. Protein concentration was measured using the Bicinchoninic acid Protein Assay Kit (Thermo Scientific). Immunoprecipitation of endogenous Prdx 5 from cell lysates was carried out using Protein G Sepharose (Generon) and anti-Prdx 5 antibody. Affinity purification of SBP-Prdx 5 was carried out using streptavidin beads (Upstate Biotechnology Inc). Proteins were eluted with $2 \times$ SDS loading buffer and analysed by anti-CoA Western blots. SDS-PAGE separated proteins were transferred to a PVDF membrane (Bio-Rad Laboratories) which was then blocked with Odyssey blocking buffer. The membrane was incubated in primary antibodies for $2 \mathrm{~h}$ at room temperature (RT) or overnight at $4{ }^{\circ} \mathrm{C}$, and with secondary antibodies for $30 \mathrm{~min}$ RT. Immunoreactive bands were visualised using Odyssey Scanner CLx and Image Studio Lite software (LI-COR Biosciences).

\section{Heart perfusion}

Sprague-Dawley rats (120-300 g) were used in this study. All experiments involving animals were performed in accordance with the European Convention for the Protection of Vertebrate Animals used for Experimental and Other Scientific Purposes (CETS no. 123) and the UK Animals (Scientific Procedures) Act 1986 amendment regulations 2012. Heart perfusion was performed as previously described, with minor modifications. BSA was omitted and $11 \mathrm{mM}$ glucose and $1.8 \mathrm{mM} \mathrm{CaCl}_{2}$ were added to Krebs-Henseleit buffer (KHB) [22]. Hearts were perfused with KHB for $10 \mathrm{~min}$ followed by $20 \mathrm{~min}$ perfusion in the presence or absence of $100 \mu \mathrm{M} \mathrm{H}_{2} \mathrm{O}_{2}$.

\section{Expression and affinity purification of Prdx5}

Escherichia coli BLR (DE3) cells were transformed with plasmid containing His-tagged human Prdx 5 coding sequence [21]. Expression and affinity purification of HisPrdx 5 on Talon Resin (Clontech Laboratories) was performed as described previously [23]. Eluted protein was dialysed against $20 \mathrm{mM}$ Tris- $\mathrm{HCl}$ (pH 7.5) containing $1 \mathrm{mM}$ EDTA and stored at $-80^{\circ} \mathrm{C}$.

\section{In vitro CoAlation assay}

Purified recombinant His-Prdx $5(0.5 \mu \mathrm{g})$ was incubated with a mixture of oxidised and reduced forms of $\mathrm{CoA}$ (CoASH and CoASSCoA, $1 \mathrm{mM}$ final) in $20 \mathrm{mM}$ Tris- $\mathrm{HCl}$, pH 7.5 for $30 \mathrm{~min}$ at RT. The mixture was passed through a BioSpin 6 column (Bio-Rad) to remove excess CoA, and this preparation of Prdx 5 was further used in activity assays. For Western blot analysis, NEM (10 mM final) was added to the samples for $10 \mathrm{~min}$ before mixing with SDS loading buffer $(1 \times$ final $)$ with or without DTT.

For SDS-PAGE analysis, $2 \mu \mathrm{g}$ of His-Prdx 5 was incubated with CoA and CoASSCoA as previously described, or with $\mathrm{H}_{2} \mathrm{O}_{2}$ (1 mM final) for 10 min or with buffer alone, and was mixed with reducing or non-reducing loading buffer, before loading on the gel.

\section{Prdx5 activity assay}

$\operatorname{Prdx} 5$ activity was measured using the thioredoxin system as described previously [24]. The rate of $\mathrm{H}_{2} \mathrm{O}_{2}$ degradation was measured by monitoring the decrease in $A_{340}$ caused by NADPH oxidation. The assay was performed in a $150 \mu \mathrm{l}$ reaction mixture, containing $50 \mathrm{mM}$ Hepes- $\mathrm{NaOH}$ (pH 7), $200 \mu \mathrm{M}$ NADPH, $760 \mathrm{nM}$ mouse TrxR, $11 \mu \mathrm{M}$ 
A

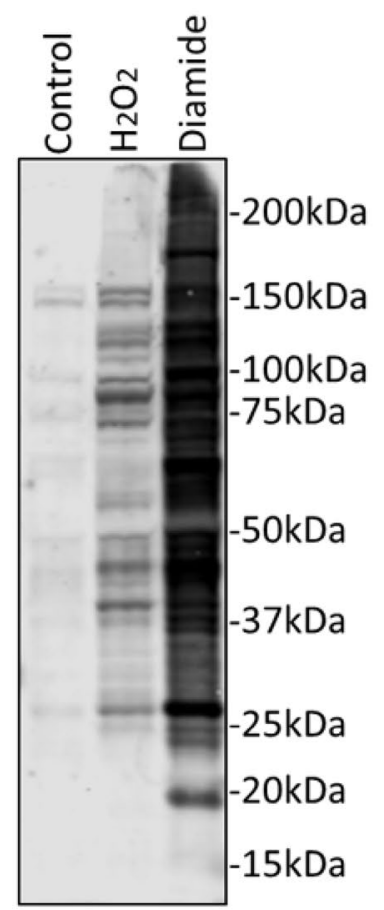

WB: anti-CoA

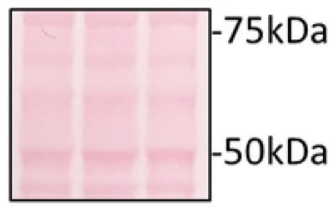

Ponceau S
B

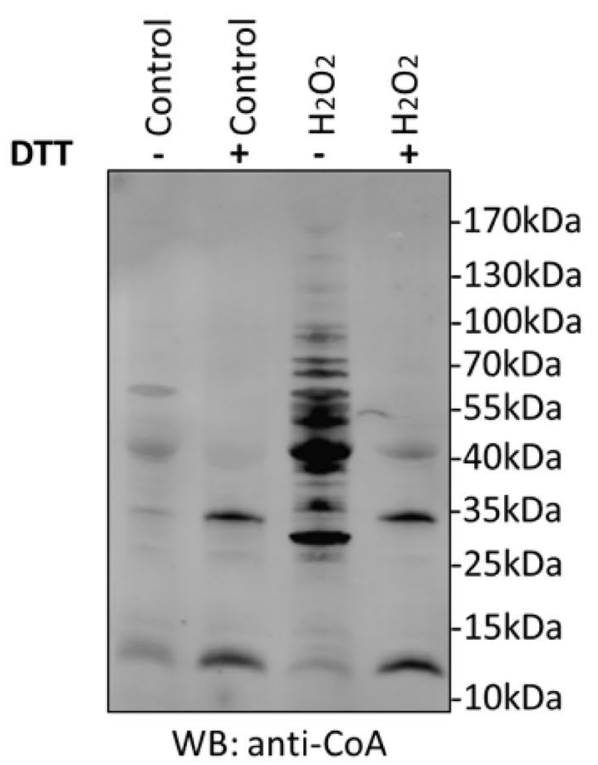

C

Prdx5

$m / z 946.94^{2+}$

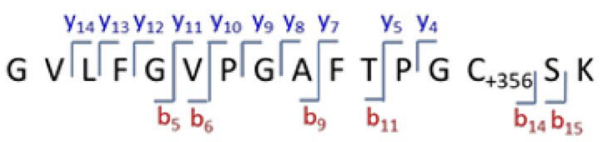

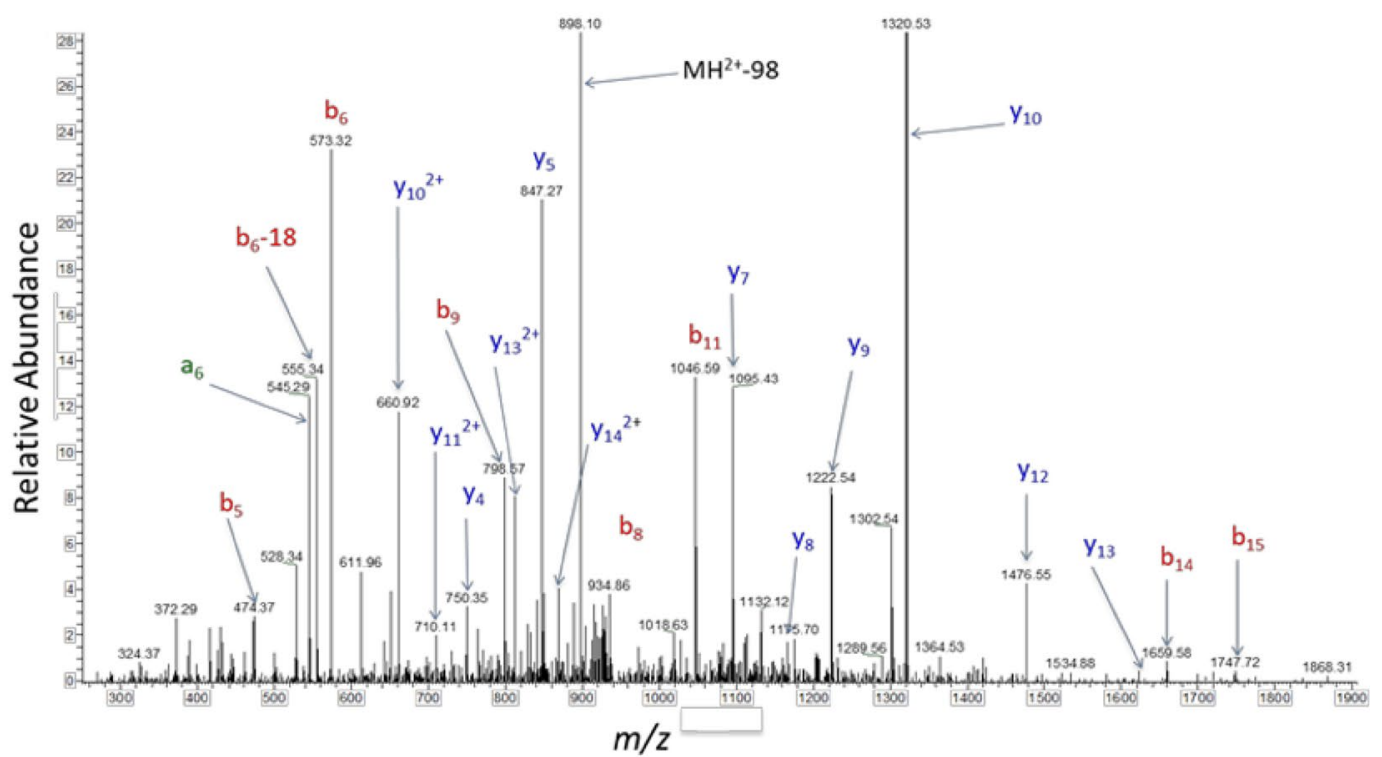


४Fig. 1 Prdx5 is CoAlated in HEK293/Pank1 $\beta$ cells and in perfused rat heart in response to oxidative stress. a Anti-CoA Western blot reveals extensive modification of cellular proteins by $\mathrm{CoA}$ in HEK293/Pank1 $\beta$ cells treated with $500 \mu \mathrm{M} \mathrm{H} \mathrm{H}_{2} \mathrm{O}_{2}$ and $500 \mu \mathrm{M}$ diamide for $30 \mathrm{~min}$. b Analysis of protein CoAlation in isolated rat hearts perfused in the presence or absence of $100 \mu \mathrm{M} \mathrm{H}_{2} \mathrm{O}_{2}$ for 20 min, $\mathrm{N}=3$. $\mathbf{c}$ LC-MS/MS spectrum of a CoAlated peptide (GVLFGVPGAFTPGCSK), corresponding to peroxiredoxin 5 (Prdx5). The Prdx5-derived peptide was immunoprecipitated with anti-CoA antibody $1 \mathrm{~F} 10$ from trypsin/LysC digested protein extracts of rat heart perfused with $100 \mu \mathrm{M} \mathrm{H}_{2} \mathrm{O}_{2}$ and analysed by LC-MS/MS as previously described (Y. Tsuchiya et al.)

human Trx and $1 \mu \mathrm{M} \operatorname{Prdx} 5$ (control or in vitro CoAlated). The mix was incubated at $37{ }^{\circ} \mathrm{C}$ for $5 \mathrm{~min}$, and the reaction initiated by the addition of $500 \mu \mathrm{M} \mathrm{H}_{2} \mathrm{O}_{2}$.

\section{Statistical analysis}

Where appropriate, values are given as means \pm SEM.

\section{Mass spectrometry and data processing}

Peptides resulting from enzymatic digestion were analysed by nano-scale capillary LC-MS/MS using an Ultimate U3000 UPLC System (Dionex) fitted with a $100 \mu \mathrm{m} \times 2 \mathrm{~cm}$ PepMap100 $\mathrm{C}_{18}$ nano trap column and a $75 \mu \mathrm{m} \times 25 \mathrm{~cm}$ PepMap100 $\mathrm{C}_{18}$ nano analytical column (Dionex). Peptides were eluted using an acetonitrile gradient and sprayed directly via a nano-flow electrospray ionisation source into the mass spectrometer (Orbitrap Velos, Thermo Scientific). The mass spectrometer was operated in data dependent mode, using a full scan $(\mathrm{m} / \mathrm{z}=350-1600)$ in the Orbitrap analyser, with a resolution of 60,000 at $\mathrm{m} / \mathrm{z}=400$, followed by MS/MS acquisitions of the 20 most intense ions in the LTQ Velos ion trap. Maximum FTMS scan accumulation times were set at $250 \mathrm{~ms}$ and maximum ion trap MSn scan accumulation times were set at $200 \mathrm{~ms}$. The Orbitrap measurements were internally calibrated using the lock mass of polydimethylcyclosiloxane at $\mathrm{m} / \mathrm{z} 445.120025$. Dynamic exclusion was set for $30 \mathrm{~s}$ with exclusion list of 500. LC-MS/MS raw data files were processed as standard samples using MaxQuant [25] version 1.5.2.8 which incorporates the Andromeda search engine. MaxQuant processed data were searched against either a Rat or Human UniProt protein database. Carbamidomethyl cysteine, Acetyl N-terminal, N-ethylmaleimide cysteine, oxidation of methionines, CoAlation of cysteine with delta mass 338, 356 and 765, were set as variable modifications. For all data sets, the default parameters in MaxQuant were used, except MS/MS tolerance, which was set at 0.6 Da and the second peptide ID was unselected.

\section{Results}

\section{Mass spectrometry-based identification of Prdx5 CoAlation}

To study the role of CoA in redox regulation in mammalian cell and tissues, we employed the HEK293/Pank $1 \beta$ cell and Langendorff-perfused heart models. HEK293/ Pank $1 \beta$ cells were grown to $60 \%$ confluency in complete Dulbecco's modified Eagle's medium (DMEM) and then cultured in pyruvate-free, low glucose DMEM for $18 \mathrm{~h}$. Oxidative stress was induced by incubating cells in the presence of $500 \mu \mathrm{M} \mathrm{H}_{2} \mathrm{O}_{2}$ or $500 \mu \mathrm{M}$ diamide for $30 \mathrm{~min}$. Protein extracts were separated on non-reducing SDSPAGE and immunoblotted with anti-CoA monoclonal antibody. As shown in Fig. 1a, only a few proteins are CoAlated at a background level in control non-treated cells, while extensive protein CoAlation is observed in response to $\mathrm{H}_{2} \mathrm{O}_{2}$ and diamide.

In addition, we used the Langendorff-perfused heart model for examining protein CoAlation in rat heart exposed to oxidative stress. In this study, isolated rat hearts were perfused with $100 \mu \mathrm{M} \mathrm{H}_{2} \mathrm{O}_{2}$ for $20 \mathrm{~min}$ and homogenised as described in Materials and Methods. We found that $\mathrm{H}_{2} \mathrm{O}_{2}$ perfusion significantly increased protein CoAlation when compared to control, and this was reversed to the level of untreated control by DTT (Fig. 1b).

Extensive protein CoAlation in response to oxidative stress in HEK293/Pank $1 \beta$ and perfused rat heart prompted us to identify CoA-modified proteins using the developed methodology [17]. In our studies, we used HEK293/Pank1 $\beta$ cells which overexpress pantothenate kinase $1 \beta$ (the main rate-limiting enzyme in CoA biosynthesis) and produce comparable levels of CoA as those found in rat heart, liver, kidney or primary cardiomyocytes. In fact, analysis showed that established cell lines HepG2 and HEK293 contain much lower concentrations of CoA when compared to those found in the previously mentioned sources [26]. A diverse range of proteins was found to be CoAlated in prepared samples from HEK293/ Pank $1 \beta$ cells and perfused rat heart. The vast majority of CoAlated proteins were found to be involved in metabolic processes (over $65 \%$ ), as well as proteins implicated in stress response. Notably, an atypical member of the peroxiredoxin family, Prdx5, was found to be CoAlated at peroxidatic Cys48 in both HEK293/Pank $1 \beta$ cells and perfused rat heart exposed to $\mathrm{H}_{2} \mathrm{O}_{2}$. Figure $1 \mathrm{c}$ shows the liquid chromatography tandem-mass spectrometry spectrum of a cysteine-containing peptide derived from Prdx 5 (GVLFGVPGAFTPGCSK) with an increase in $356 \mathrm{Da}$, corresponding to covalently attached 4-phosphopantetheine to cysteine residue. 


\section{Prdx5 CoAlation is induced in cells by a panel of oxidising agents}

Induction of Prdx 5 CoAlation by $\mathrm{H}_{2} \mathrm{O}_{2}$ in both experimental models prompted us to examine whether $\operatorname{Prdx} 5$ is $\mathrm{CoA}$ lated in cellular response to other oxidising agents. In this study, cells were transfected with pCR3.1-MAR/wt-Prdx 5 plasmid, which drives the expression of human Prdx 5 with the $\mathrm{N}$-terminally tagged streptavidin binding peptide (SBP). Transfected cells were grown in pyruvate-free and low glucose media for $18 \mathrm{~h}$ and then treated with or without $500 \mu \mathrm{M} \mathrm{H}_{2} \mathrm{O}_{2}, 500 \mu \mathrm{M}$ diamide or $50 \mu \mathrm{M}$ menadione for $30 \mathrm{~min}$ to induce oxidative stress. Transiently overexpressed SBP-Prdx 5 was pulled down from cell lysates using streptavidin beads and both total and the pulled down proteins were analysed by SDS-PAGE under non-reducing conditions followed by immunoblotting with anti-CoA antibody. As shown in Fig. 2a, treatment of cells with $\mathrm{H}_{2} \mathrm{O}_{2}$, diamide and menadione induces extensive modification of cellular proteins by CoA in examined total protein extracts. Weak background immunoreactive signal was observed in control untreated cells. Immunoblotting of pulled down SBP-Prdx5 revealed strong induction of Prdx 5 CoAlation in cells treated with all oxidising agents, and the highest level is observed in response to diamide treatment (Fig. 2b).

To further validate the above findings, we examined CoAlation of endogenous Prdx 5 from HEK293/Pank1 $\beta$ cells or primary rat cardiomyocytes treated or untreated with $500 \mu \mathrm{M}$ diamide for 30 min. Endogenous Prdx 5 was immunoprecipitated from total cell lysates with anti-Prdx 5 antibody and the immune complexes probed with anti-CoA and anti-Prdx 5 antibodies. The induction of endogenous Prdx 5 CoAlation in response to diamide treatment is shown in Fig. 2c, d.

\section{Prdx5 CoAlation is induced by glucose deprivation}

Glucose is the primary source of energy in most eukaryotic cells and glucose deprivation causes metabolic oxidative stress. The observation that culturing HEK293/Pank1 $\beta$ cells in pyruvate-free and low glucose medium resulted in subtle increase of CoAlated Prdx5 (Fig. 2c), led us to examine the effect of metabolic stress induced by glucose deprivation on Prdx 5 CoAlation. Here, HEK293/Pank1 $\beta$ cells were transfected with pCR3.1-MAR/wt-Prdx5 plasmid and, $24 \mathrm{~h}$ later, the transfection medium was replaced with pyruvateand glucose-free DMEM. Cells were harvested after $18 \mathrm{~h}$ and total protein extracts and streptavidin-Sepharose pulled down proteins were analysed by immunoblotting with antiCoA antibody.

As shown in Fig. 3a, significant increase in protein CoAlation was observed in cells cultured in glucose-deprived media. Notably, adding full media back to metabolically
A

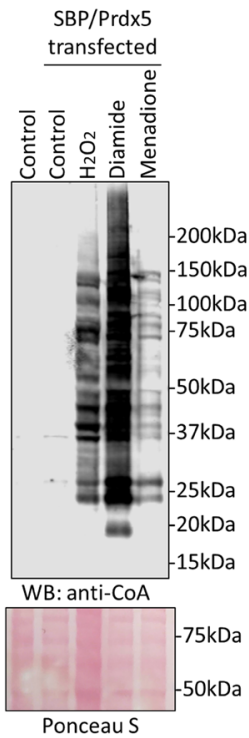

C

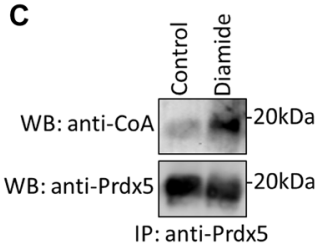

B

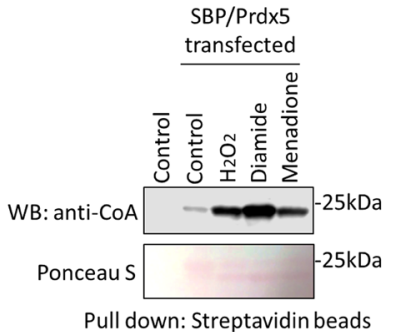

D

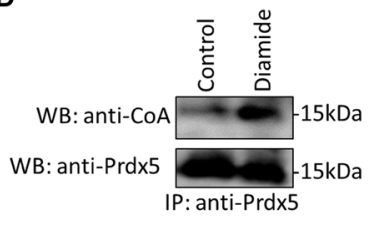

Fig. 2 CoAlation of endogenous and transiently overexpressed Prdx 5 is induced in response to oxidising agents. a Western blot analysis of protein CoAlation in HEK293/Pank1 $\beta$ cells treated for 30 min with diamide $(500 \mu \mathrm{M})$, menadione $(50 \mu \mathrm{M})$ or $\mathrm{H}_{2} \mathrm{O}_{2}(500 \mu \mathrm{M})$. b Transiently overexpressed SBP-tagged Prdx 5 was pulled down from cell lysates and bound proteins examined by immunoblotting with antiCoA antibodies. c Western blot analysis of immunoprecipitated endogenous Prdx 5 from HEK293/Pank1 $\beta$ cells or primary cardiomyocytes $\mathbf{d}$ treated or not treated with $500 \mu \mathrm{M}$ diamide for $30 \mathrm{~min}$. $\mathrm{N}=3$

stressed cells for 30 min resulted in significant de-CoAlation of cellular proteins, which was almost at the level of control cells. Immunoblotting of streptavidin-Sepharose pull-down complexes with anti-CoA antibodies revealed markedly increased CoAlation of SBP-Prdx5 in glucose-deprived cells (Fig. 3b). A background level of SBP-Prdx 5 CoAlation was observed in control cells. To find out whether the induction of SBP-Prdx 5 CoAlation by glucose deprivation is a reversible post-translational modification, complete media was re-introduced for $30 \mathrm{~min}$. As shown in Fig. 3b, glucose deprivation-induced Prdx 5 CoAlation is completely reversed after the re-addition of complete DMEM medium.

\section{Mutational analysis of Prdx5 CoAlation in response to oxidative and metabolic stress}

Prdx 5 possesses three cysteine residues: Cys48 and Cys 152 correspond to peroxidatic and resolving cysteines respectively, while Cys73 is not involved in the catalytic 
A

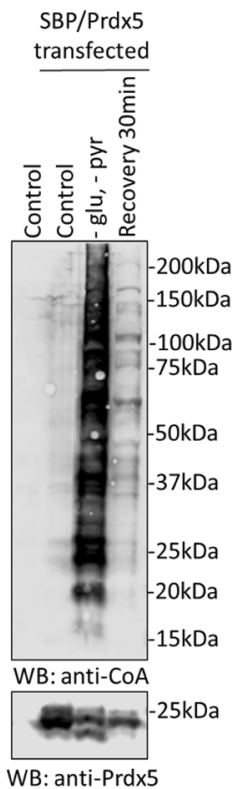

Fig. 3 CoAlation of transiently overexpressed Prdx5 in HEK293/ Pank $1 \beta$ cells is induced by metabolic stress. HEK293/Pank1 $\beta$ cells were grown in pyruvate and glucose-free media for $18 \mathrm{~h}$ to induce metabolic stress. To find whether Prdx5 CoAlation is a reversible post-translational modification, complete media was then re-introduced for $30 \mathrm{~min}$ (recovery lane). Total protein (a) or SBP-Prdx5 (b) CoAlation was analysed by anti-CoA immunoblot. $\mathrm{N}=3$

mechanism [23]. The LC-MS/MS analysis revealed CoAlation of peroxidatic Cys48 in HEK293/Pank1 $\beta$ cells and perfused rat heart exposed to $\mathrm{H}_{2} \mathrm{O}_{2}$. To validate this finding and investigate whether other cysteine residues are covalently modified by $\mathrm{CoA}$ under oxidative and metabolic stress, we carried out mutational studies using a panel of hPrdx 5 mutants (C48S, C152S or C48/152S). Initially, we examined at which site/sites Prdx5 is CoAlated under oxidative stress. Here, HEK293/Pank $1 \beta$ cells were transfected with pCR3.1-MAR plasmids, expressing wild type or generated mutants of Prdx5. $24 \mathrm{~h}$ after transfection, cells were incubated for $30 \mathrm{~min}$ with or without $500 \mu \mathrm{M}$ diamide. Streptavidin pull-downs from lysed cells were analysed by Western blotting with anti-CoA and anti-Prdx 5 antibodies. The anti-Prdx 5 blot shows a comparable amount of pulled down SBP-Prdx 5 in all transfected cells. Diamide-induced CoAlation of wtPrdx 5 is readily detected in anti-CoA blot of streptavidin pull-downs (Fig. $4 \mathrm{a}$ and $\mathrm{b}$ ). The C48S and C152S mutants were also CoAlated, but at a reduced level, suggesting CoAlation of both peroxidatic and resolving cysteines in cellular response to oxidative stress. This assumption was confirmed when we examined diamideinduced CoAlation of the C48/152S double mutant. As shown in Fig. 4b, the C48/152S double mutant is not CoAlated in control and diamide-treated cells. These findings also indicate that the non-catalytic Cys 73 is not CoAlated in cells exposed to oxidising agents.

We also investigated CoAlation of transiently overexpressed wild type and cysteine mutants of SBP-Prdx 5 in HEK293/Pank1 $\beta$ cells under metabolic stress induced by glucose deprivation. Immunoblotting of streptavidin-Sepharose pulled down proteins with anti-CoA antibody showed background CoAlation in control cells (Fig. 4c and d). As expected, significant CoAlation of transiently expressed wtPrdx 5 was detected in glucose-deprived cells. Similar to diamide-treated cells, CoAlation of the C48S and C152S mutants was significantly lower, when compared to wtPrdx 5 (Fig. 4c and d). No CoAlation of the C48/152S double mutant was observed in glucose-deprived cells.

\section{Peroxidase activity of Prdx5 is inhibited by covalent binding of $\mathrm{CoA}$ in vitro}

We have recently developed an in vitro CoAlation assay, which allows covalent CoA modification of purified recombinant and endogenous proteins with high efficiency [17]. Using this assay, we showed that in vitro CoAlation of several enzymes from different metabolic pathways, including creatine kinase (CK) and glyceraldehyde-3-phosphate dehydrogenase (GAPDH), results in significant inhibition of their enzymatic activities [17].

To test the effect of CoAlation on Prdx 5 peroxidase activity, recombinant His-Prdx5 was incubated with or without a mix of CoASH (reduced) and CoASSCoA (oxidised) in the CoAlation buffer for $30 \mathrm{~min}$. Then, the reaction mixture was passed through a BioSpin column to remove the excess of reduced and oxidised CoA. The efficiency of in vitro CoAlation was confirmed by Western blotting with anti-CoA antibody. As shown on Fig. 5a, recombinant His-Prdx5 was modified by $\mathrm{CoA}$ in vitro in a DTT-sensitive manner. Prdx 5 activity towards $\mathrm{H}_{2} \mathrm{O}_{2}$ was then assayed by measuring the decrease in NADPH absorbance at $340 \mathrm{~nm}$ as described in M\&M. Figure $5 b$ demonstrates that in vitro CoAlation of His-Prdx 5 results in near complete inhibition (98.7\%) of the peroxidase activity. Furthermore, the inhibitory effect of CoAlation on Prdx 5 activity was efficiently reversed by the addition of $100 \mathrm{mM}$ DTT to the reaction mix. These data indicate that covalent modification of catalytic cysteines by CoA results in the inhibition of $\operatorname{Prdx} 5$ peroxidase activity and the inhibitory effect is reversed by the reducing power of DTT.

We also examined electrophoretic mobility of control (untreated), oxidised $\left(\mathrm{H}_{2} \mathrm{O}_{2}\right.$ treated) and CoAlated HisPrdx 5 separated under reducing and non-reducing conditions. This analysis showed that control, oxidised and CoAlated His-Prdx 5 run as a single band of $\sim 20 \mathrm{kDa}$ under reducing conditions (Fig. 5c). However, different pattern of electrophoretic mobility was observed under non-reducing 
Fig. 4 Mutational analysis of Prdx5 CoAlation in cellular response to oxidative and metabolic stress. a HEK293/ Pank1 $\beta$ cells transfected with wild type (WT) and C48S mutant of SBP-Prdx 5 were incubated with and without $500 \mu \mathrm{M}$ diamide for $30 \mathrm{~min}$; b HEK293/Pank1 $\beta$ cells transfected with wild type, C152S and C48/152S mutants of SBP-Prdx 5 were incubated with or without $500 \mu \mathrm{M}$ diamide for 30 min. c, d HEK293/Pank1 $\beta$ cells transfected with wild type and mutants of SBP-Prdx 5 were grown in pyruvate (pyr) and glucose (glu) free media for $18 \mathrm{~h}$ to induce metabolic stress. Transiently overexpressed SBP-tagged WT Prdx5, C48S, C152S and C48/152S mutants were pulled down with Streptavidin beads and examined by anti-CoA immunoblot. $\mathrm{N}=3$

A

B

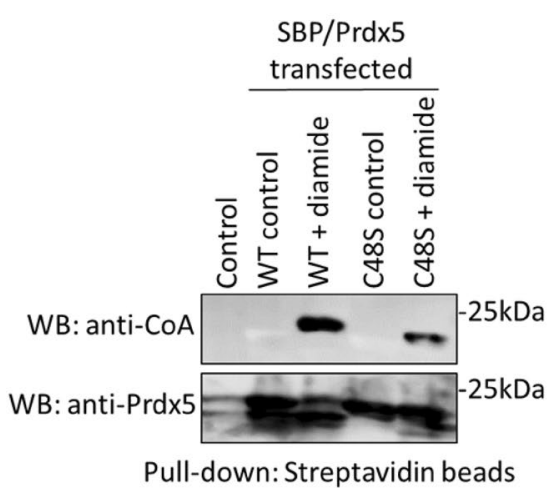

C

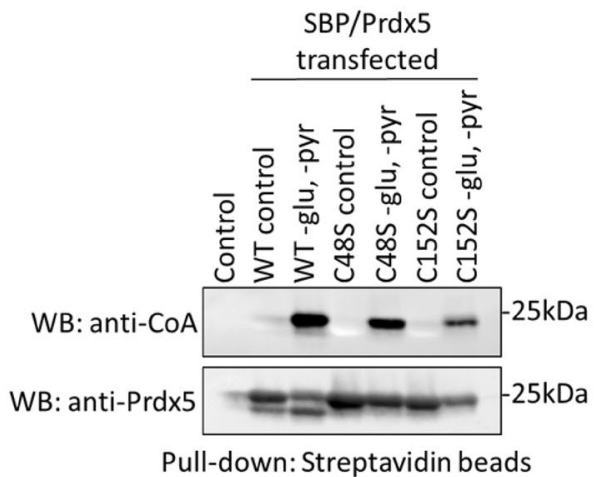

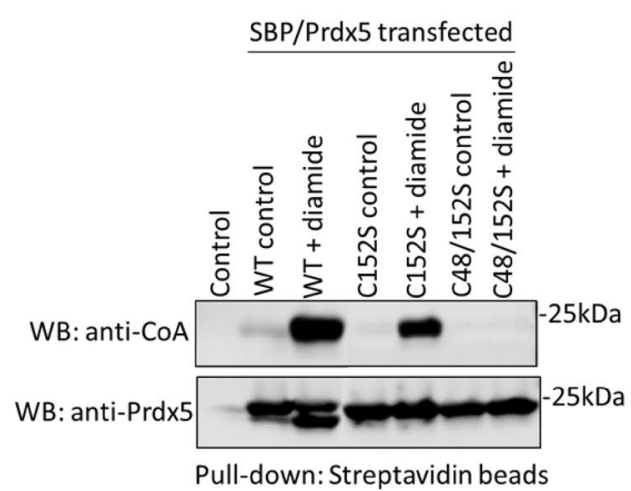

D

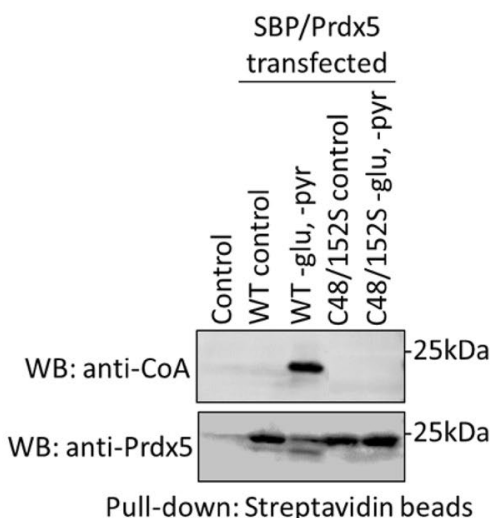

conditions. In vitro CoAlated Prdx5 showed the same mobility under reducing and non-reducing conditions $(\sim 20 \mathrm{kDa})$. The exposure of recombinant His-Prdx 5 to $\mathrm{H}_{2} \mathrm{O}_{2}$ results in faster mobility $(\sim 18 \mathrm{kDa})$ under non-reducing conditions, suggesting significant conformational changes in a monomeric form of Prdx 5 mediated by a disulphide bond formation. These findings are in agreement with published crystallographic studies of reduced and oxidised forms of $\operatorname{Prdx} 5$ $[23,24]$. The distance between the two sulphur atoms of peroxidatic Cys 48 and resolving Cys152 in a reduced form of $\operatorname{Prdx} 5$ is $13.8 \mathrm{~A}^{\circ}$ [24]. The overall fold of oxidised Prdx 5 is very similar to that of the reduced form and the main conformational changes involve the movement of the regions containing peroxidatic and resolving cysteines, allowing the formation of a disulphide bridge and a more compact fold [23]. A significant proportion of control His-Prdx5, which was stored at $-20^{\circ} \mathrm{C}$ in buffer without DTT, also runs with higher electrophoretic mobility. These data indicate that recombinant Prdx5 is prone to oxidation when stored without DTT even for a short period of time. It has been reported that freshly grown Prdx 5 crystals can only survive for a few days when they are simply exposed to air, whereas crystals of the $\mathrm{C} 48 \mathrm{~S}$ mutant are much more stable, even in presence of $\mathrm{H}_{2} \mathrm{O}_{2}$ [24].
It has been reported that PRDX5 can also form dimers, known as type A dimers, and most contacts involve hydrophobic interactions with the exception of an inter-molecular salt bridge between Arg 124 and Asp77 [23]. A minor percentage of dimeric $(\sim 40 \mathrm{kDa})$ and potentially trimeric $(\sim 60 \mathrm{kDa})$ forms of Prdx 5 was observed under non-reducing conditions, and their appearance is DTT-dependent (Fig. 5c).

\section{Discussion}

$\operatorname{Prdx} 5$ was the last mammalian isoform of the Prdx family to be identified and therefore, the knowledge on its regulation by signalling pathways via binding partners and post-translational modifications is lagging. To date, several post-translational modifications of Prdx5, including phosphorylation, acetylation and glutathionylation, have been reported in proteome-wide studies, but their physiological relevance remains unclear [27-29]. Interestingly, glutathionylation of Prdx 5 was detected by MALDI-MS in primary rat hepatocytes exposed to oxidative stress. However, the site of glutathionylation and significance of this modification in the regulation of $\operatorname{Prdx} 5$ catalytic activity has yet to be investigated [28]. 
A

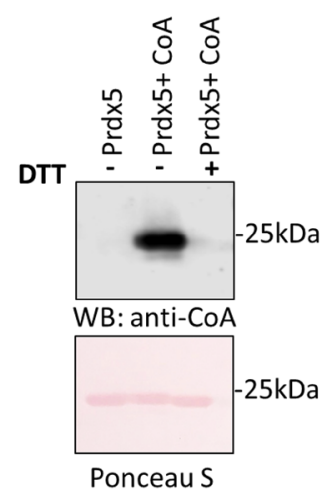

C

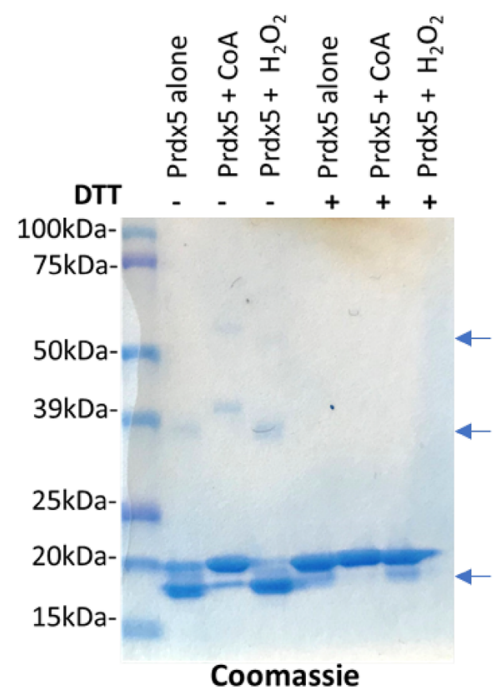

Fig. 5 Regulation of the Prdx5 enzymatic cycle by CoAlation a In vitro CoAlted-Prdx 5 was boiled in loading buffer with or without DTT. CoAlation of Prdx 5 was examined by anti-CoA immunoblot. b NADPH oxidation coupled to the peroxidase activity of Prdx5 in the presence of the Trx systems. The initial rate of NADPH oxidation was monitored by measurement of the decrease in $A_{340}$ in the presence of $\operatorname{Prdx} 5$ at $37{ }^{\circ} \mathrm{C}$. The $150 \mu \mathrm{l}$ reaction mixture contained $50 \mathrm{mM}$ Hepes- $\mathrm{NaOH}$ (pH 7), $200 \mu \mathrm{M}$ NADPH, $76 \mathrm{nM}$ mouse TrxR, $1.1 \mu \mathrm{M}$ human Trx, and $2 \mu \mathrm{g}$ untreated Prdx5 (control), in vitro CoAlated Prdx 5 or in vitro CoAlated Prdx 5 with DTT. The reaction was initiated by the addition of $0.5 \mathrm{mM} \mathrm{H}_{2} \mathrm{O}_{2}$. c Recombinant $\operatorname{Prdx} 5$ was

In this paper, we report for the first-time covalent modification of Prdx 5 by $\mathrm{CoA}$ in cellular response to oxidative and metabolic stress. Evidence is provided that Prdx 5 is CoAlated at low background level in exponentially growing cells, while exposure to oxidising agents induces covalent modification of transiently overexpressed and endogenous $\operatorname{Prdx} 5$ by CoA. Furthermore, metabolic stress induced by glucose deprivation also leads to a significant increase in Prdx 5 CoAlation, which is efficiently reversed by the readdition of glucose to the culture medium. These findings

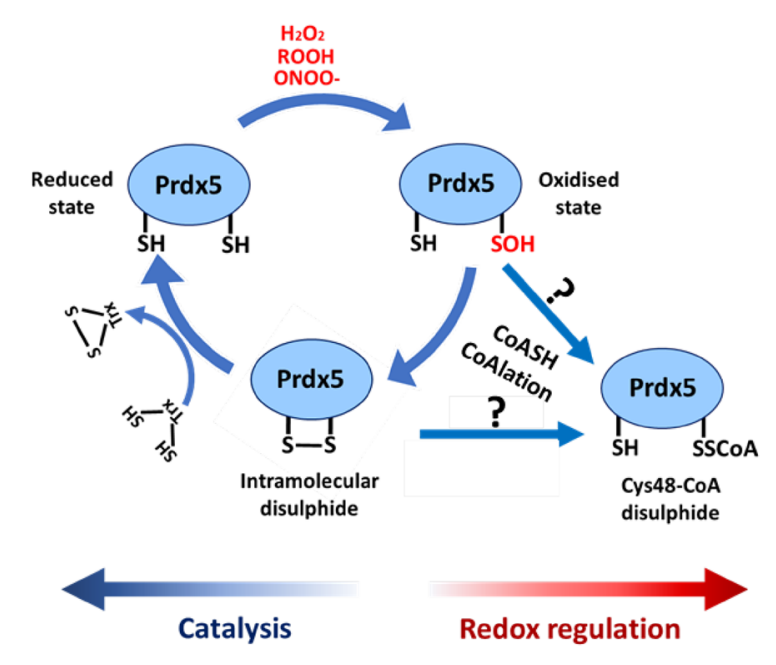

incubated with oxidised and reduced $\mathrm{CoA}$ or with $\mathrm{H}_{2} \mathrm{O}_{2}$ for $30 \mathrm{~min}$, or with buffer (Prdx5 alone). The samples were separated by SDSPAGE under reducing (+DTT), and non-reducing conditions (-DTT). The arrows indicate the position of monomeric and dimeric forms of His-Prdx5. d Schematic representation of the Prdx5 catalytic cycle. In the presence of a substrate molecule, Prdx 5 peroxidatic cysteine is oxidised to sulphenic acid (Cys-SOH) and condenses to form an intramolecular disulphide with the resolving cysteine. In prolonged oxidative or metabolic stress conditions, the CoA thiol attacks the cysteine sulphenic acid, or disulphide bond and binds to catalytic Cys48

are in agreement with our recently published studies on redox regulation of protein CoAlation in prokaryotic and eukaryotic cells $[17,18]$.

Human Prdx5 and orthologues in vertebrates and invertebrates possess three highly conserved cysteine residues: Cys48, Cys73 and Cys152. Crystallographic studies of hPrdx 5 in reduced and oxidised states revealed that both peroxidatic Cys48 and resolving Cys 152 are surface exposed, while Cys 73 is completely buried [23, 24]. The LC-MS/MS analysis of CoAlated proteins in HEK293/Pank1 $\beta$ cells and 
perfused rat hearts exposed to $\mathrm{H}_{2} \mathrm{O}_{2}$ revealed CoAlation of only the peroxidatic Cys 48 . The peroxidatic cysteines are more nucleophilic and highly sensitive to attack by reactive oxygen and reactive nitrogen species, and therefore are more prone to regulatory S-thiolation, than resolving cysteine residues. The peroxidatic Cys48 in hPrdx 5 has a low pKa (5.2), making it deprotonated at physiological $\mathrm{pH}$ and more nucleophilic [29]. In normal metabolic conditions, the peroxidatic cysteine residue of $\operatorname{Prdx} 5$ reduces a peroxide substrate and is oxidised to sulphenic acid. The resolving Cys 152 then attacks this cysteine sulphenic acid forming an intramolecular disulphide bond, which is reduced by thioredoxin to regenerate an active enzyme. Thioredoxin reductase reduces the oxidised thioredoxin, using NADPH as a reductant. In conditions of severe oxidative stress or prolonged metabolic stress, NADPH is depleted and therefore the NADPH-dependent recycling system for Prdx 5 might not function efficiently to reduce the intramolecular disulphide bond of oxidised Prdx 5. We hypothesise that the CoA thiol attacks the Cys48 cysteine sulphenic acid, or the disulphide bond formed between the peroxidatic Cys 48 and the resolving Cys152, and binds to protect the peroxidatic Cys48 from overoxidation. Indeed, it was shown in previous studies that treatment of cells with $100-200 \mu \mathrm{M}$ peroxides (t-butyl hydroperoxide, $\mathrm{H}_{2} \mathrm{O}_{2}$ ) is sufficient to cause overoxidation of Prdxs [30-32]. CoAlation of Prdx5 might be a regulatory event to protect the catalytic Cys 48 from irreversible overoxidation. In mutational studies we observed oxidative and metabolic stress-induced CoAlation of both peroxidatic Cys 48 and resolving Cys152. We speculate that in wild type Prdx5, peroxidatic Cys48 is a preferred target for the oxidation-mediated covalent modification by CoA, while CoAlation of resolving Cys 152 is a specific attribute of the Prdx5C48S mutant. Covalent modification of peroxidatic Cys 48 by CoA implies the inhibitory effect of CoAlation on Prdx 5 peroxidase activity. Indeed, in vitro CoAlated recombinant Prdx 5 was found to be enzymatically inactive and the peroxidase activity was recovered by DTT-mediated dissociation of CoA. Our studies in metabolic stress showed that CoAlation of Prdx 5 is physiologically reversible, and we have proposed the existence of CoA-redoxins that would act to reduce CoA-modified proteins [33].

These results are in agreement with our previous studies, in which we demonstrated that in vitro CoAlation of catalytically active cysteines in several metabolic enzymes, including CK, pyruvate dehydrogenase kinase 2 (PDK2) and GAPDH, impedes their catalytic activities and the inhibition is DTT-sensitive [17]. Furthermore, we have also demonstrated that in vitro CoAlation of Staphylococcus aureus GAPDH not only inhibits the enzymatic activity, but also protects the catalytic Cys 151 from overoxidation by $\mathrm{H}_{2} \mathrm{O}_{2}$ [18]. The question which remains to be answered is whether redox-induced Prdx5 CoAlation serves to protect catalytic cysteines from overoxidation and to upregulate the antioxidant defence via redox signalling. Recently, glutathionylation of $\operatorname{Prdx} 2$ under oxidative stress conditions was shown to protect the catalytic cysteines from hyperoxidation and play a role in redox signalling $[34,35]$.

The attachment of pantetheine and 3'5'-ADP moieties to CoA-modified cysteines in oxidative stress response may generate a unique binding motif for intra- and inter-molecular interactions, especially for proteins containing the nucleotide binding fold. It has been recently reported that redox-mediated modification of Prdx 1 by GSH induces its interaction with phosphatase and tensin homolog (PTEN) and mammalian Ste20-like kinase-1 (MST1) in the regulation of pro-survival signalling and the cell cycle respectively $[36,37]$. We speculate that $\operatorname{Prdx} 5$ CoAlation in cellular response to oxidative and metabolic stress may promote the formation or dissociation of regulatory complexes, which are involved in redox signalling and antioxidant defence. Recently, specific interaction between superoxide dismutase 1 (SOD1) and $\operatorname{Prdx} 5$ was shown to be critical for maintaining mitochondrial redox homeostasis and avoiding cell death [38]. It will be interesting to investigate whether the SOD1/Prdx5 interaction is affected by covalent modification of $\operatorname{Prdx} 5$ catalytic cysteines in cellular response to oxidative and metabolic stress.

Taken together, the findings presented in this study permit us to propose that the $\operatorname{Prdx} 5$ catalytic cycle operates when permissible levels of $\mathrm{H}_{2} \mathrm{O}_{2}$ are produced in cells under oxidative stress (Fig. 5c). Further accumulation of $\mathrm{H}_{2} \mathrm{O}_{2}$ can induce $\operatorname{Prdx} 5$ CoAlation, which may function to protect catalytic cysteines from overoxidation and initiate redox signalling pathways involved in antioxidant defences and the repair of oxidative damage.

Acknowledgements We thank the members of Cell Regulation laboratory at the Department of Structural and Molecular Biology (UCL) for their valuable inputs throughout this study, and UCL Darwin Research Facility for tissue culture and analytical biochemistry support.

Author contributions Conceptualization by I.G. and J.B.; B.Y., D.S., L.A., O. M., L.P. and J.B. performed cell-based experiments and in vitro CoAlation assays; S.K. and S-H. A. carried out Prdx5 enzymatic assays; S.Y.P-C. and M.S. designed and performed the MSMS experiments; J.B., B.Y., T-H. L., M.S., V. F. and I.G. designed the experiments and I.G. wrote the manuscript with input from other authors.

Funding This study was supported by BBSRC (BB/L010410/1), National Academy of Sciences of Ukraine (0110U000692) and the National Research Foundation of Korea (NRF-2017R1A2B2005938). We thank the members of Cell Regulation laboratory (UCL) for their valuable inputs throughout this study. 


\section{Compliance with ethical standards}

Conflict of interests The authors declare that they have no conflicts of interest associated with the manuscript.

Open Access This article is distributed under the terms of the Creative Commons Attribution 4.0 International License (http://creativeco mmons.org/licenses/by/4.0/), which permits unrestricted use, distribution, and reproduction in any medium, provided you give appropriate credit to the original author(s) and the source, provide a link to the Creative Commons license, and indicate if changes were made.

\section{References}

1. Rhee SG, Kil IS (2017) Multiple functions and regulation of mammalian peroxiredoxins. Annu Rev Biochem 86:749-775. https:// doi.org/10.1146/annurev-biochem-060815-014431

2. Perkins A, Nelson KJ, Parsonage D et al (2015) Peroxiredoxins: guardians against oxidative stress and modulators of peroxide signaling. Trends Biochem Sci 40:435-445. https://doi. org/10.1016/j.tibs.2015.05.001.Peroxiredoxins

3. Fischer F, Leipelt M, Wolters D, Steegborn C (2009) Identification of Peroxiredoxin 1 as a novel interaction partner for the lifespan regulator protein p66Shc. Aging (Albany NY) 1:254-265

4. Kang DH, Lee DJ, Lee S et al (2017) Interaction of tankyrase and peroxiredoxin II is indispensable for the survival of colorectal cancer cells. Nat Commun 8:1-14. https://doi.org/10.1038/s4146 7-017-00054-0

5. Sorokina EM, Feinstein SI, Zhou S et al (2011) Intracellular targeting of peroxiredoxin 6 to lysosomal organelles requires MAPK activity and binding to 14-3-3 $\varepsilon$. Am J Physiol Cell Physiol 6:1430-1441. https://doi.org/10.1152/ajpcell.00285.2010

6. Nguyên-nhu NT, Berck J, Clippe A et al (2007) Human peroxiredoxin 5 gene organization, initial characterization of its promoter and identification of alternative forms of mRNA. Biochim Biophys Acta Gene Struct Expr 1769:472-483. https://doi. org/10.1016/j.bbaexp.2007.05.004

7. Knoops B, Goemaere J, Van der Eecken V, Declercq JP (2011) Peroxiredoxin 5: structure, mechanism, and function of the mammalian atypical 2-cys peroxiredoxin. Antioxid Redox Signal 15:818-829. https://doi.org/10.1089/ars.2013.5179

8. Barranco-Medina S, Lázaro JJ, Dietz KJ (2009) The oligomeric conformation of peroxiredoxins links redox state to function. FEBS Lett 583:1809-1816

9. Radyuk SN, Michalak K, Klichko VI et al (2009) Peroxiredoxin 5 confers protection against oxidative stress and apoptosis and also promotes longevity in Drosophila. Biochem J 419:437-445. https ://doi.org/10.1042/BJ20082003

10. Leonardi R, Zhang YM, Rock CO, Jackowski S (2005) Coenzyme A: back in action. Prog Lipid Res 44:125-153. https://doi. org/10.1016/j.plipres.2005.04.001

11. Davaapil H, Tsuchiya Y, Gout I (2014) Signalling functions of coenzyme A and its derivatives in mammalian cells. Biochem Soc Trans 42:1056-1062. https://doi.org/10.1042/BST20140146

12. Srinivasan B, Sibon OCM (2014) Coenzyme A, more than "just" a metabolic cofactor. Biochem Soc Trans 42:1075-1079

13. Theodoulou FL, Sibon OCM, Jackowski S, Gout I (2014) Coenzyme A and its derivatives: renaissance of a textbook classic. Biochem Soc Trans 42:1025-1032. https://doi.org/10.1042/BST20 140176
14. Mcallister RA, Fixter LM, Campbell EHG (1988) The effect of tumour growth on liver pantothenate, CoA, and fatty acid synthetase activity in the mouse. Br J Cancer 57:83-86

15. Brass EP, Tahiliani AG, Allen RH, Stabler SP (1990) Coenzyme a metabolism in vitamin B-12 —deficient rats. J Nutr 120:290-297

16. Reibel DK, Wyse BW, Berkich DA, Neely JR (1981) Regulation of coenzyme A synthesis effects of diabetes and fasting in heart muscle: effects of diabetes and fasting. Am J Physiol 240:H606

17. Tsuchiya Y, Peak-Chew SY, Newell C et al (2017) Protein CoAlation: a redox-regulated protein modification by coenzyme A in mammalian cells. Biochem J 474:2489-2508. https://doi. org/10.1042/BCJ20170129

18. Tsuchiya Y, Zhyvoloup A, Baković J et al (2018) Protein CoAlation and antioxidant function of coenzyme $\mathrm{A}$ in prokaryotic cells. Biochem J 475(11):1909-1937

19. Malanchuk OM, Panasyuk GG, Serbin NM et al (2015) Generation and characterization of monoclonal antibodies specific to coenzyme A. Biopolym Cell 31:187-192

20. Chouchani ET, Pell VR, Gaude E et al (2014) Ischaemic accumulation of succinate controls reperfusion injury through mitochondrial ROS. Nature 515:431

21. Choi HI, Chung KJ, Yang HY et al (2013) Peroxiredoxin v selectively regulates IL- 6 production by modulating the Jak2Stat5 pathway. Free Radic Biol Med 65:270-279. https://doi. org/10.1016/j.freeradbiomed.2013.06.038

22. Clark H, Carling D, Saggerson D (2004) Covalent activation of heart AMP-activated protein kinase in response to physiological concentrations of long-chain fatty acids. Eur J Biochem 271:2215-2224. https://doi.org/10.111 1/j.1432-1033.2004.04151.x

23. Smeets A, Marchand C, Linard D, Knoops B, Declercq JP (2008) The crystal structures of oxidized forms of human peroxiredoxin 5 with an intramolecular disulfide bond confirm the proposed enzymatic mechanism for atypical 2-cys peroxiredoxins. Arch Biochem Biophys 477(98-104):2008. https://doi. org/10.1016/j.abb.2008.04.036

24. Seo MS, Kang SW, Kim K et al (2000) Identification of a new type of mammalian peroxiredoxin that forms an intramolecular disulfide as a reaction intermediate. J Biol Chem 275:2034620354. https://doi.org/10.1074/jbc.M001943200

25. Cox J, Mann M (2008) MaxQuant enables high peptide identification rates, individualized p.p.b.-range mass accuracies and proteome-wide protein quantification. Nat Biotechnol 26:1367-1372

26. Tsuchiya Y, Peak-Chew SY, Newell C et al (2017) Protein CoAlation: a redox-regulated protein modification by coenzyme A in mammalian cells. Biochem J 474(14):2489. https://doi. org/10.1042/BCJ20170129

27. Nguyen TTM, Wong R, Menazza S et al (2014) Cyclophilin D modulates the mitochondrial acetylome Tiffany. Circ Res 113:1308-1319. https://doi.org/10.1161/CIRCRESAHA 113.301867.Cyclophilin

28. Fratelli M, Demol H, Puype M et al (2003) Identification of proteins undergoing glutathionylation in oxidatively stressed hepatocytes and hepatoma cells. Proteomics 3:1154-1161. https://doi. org/10.1002/pmic.200300436

29. Zemskova M, Ling J, Lilly M (2007) PIM1 kinase protects cells from death induced by oxidative stress: potential role of PRDX5 phosphorylation. Cancer Res 67:3747

30. Yang KS, Kang SW, Woo HA et al (2002) Inactivation of human peroxiredoxin I during catalysis as the result of the oxidation of the catalytic site cysteine to cysteine-sulfinic acid. J Biol Chem 277:38029-38036. https://doi.org/10.1074/jbc.M206626200

31. Rabilloud T, Heller M, Gasnier F et al (2002) Proteomics analysis of cellular response to oxidative stress. evidence for in vivo 
overoxidation of peroxiredoxins at their active site. J Biol Chem 277:19396-19401. https://doi.org/10.1074/jbc.M106585200

32. So YK, Jo HY, Mi HK et al (2008) $\mathrm{H}_{2} \mathrm{O}_{2}$-dependent hyperoxidation of peroxiredoxin 6 (Prdx6) plays a role in cellular toxicity via up-regulation of iPLA2 activity. J Biol Chem 283:33563-33568. https://doi.org/10.1074/jbc.M806578200

33. Gout I (2018) Coenzyme A, protein CoAlation and redox regulation in mammalian cells. Biochem Soc Trans 46:721-728. https ://doi.org/10.1042/BST20170506

34. Peskin AV, Pace PE, Behring JB et al (2016) Glutathionylation of the active site cysteines of peroxiredoxin 2 and recycling by glutaredoxin. J Biol Chem 291:3053-3062. https://doi.org/10.1074/ jbc.M115.692798

35. Netto LES, Antunes F (2016) The roles of peroxiredoxin and thioredoxin in hydrogen peroxide sensing and in signal transduction. Mol Cells 39:65-71
36. Morinaka A, Funato Y, Uesugi K, Miki H (2011) Oligomeric peroxiredoxin-I is an essential intermediate for $\mathrm{p} 53$ to activate MST1 kinase and apoptosis. Oncogene 30:4208-4218. https:// doi.org/10.1038/onc.2011.139

37. Cao J, Schulte J, Knight A et al (2009) Prdx1 inhibits tumorigenesis via regulating PTEN/AKT activity. EMBO J 28:1505-1517

38. Malty RH, Aoki H, Kumar A et al (2017) A map of human mitochondrial protein interactions linked to neurodegeneration reveals new mechanisms of redox homeostasis and NF- $\mathrm{KB}$ signaling. Cell Syst 5:564-577.e12. https://doi.org/10.1016/j.cels.2017.10.010

Publisher's Note Springer Nature remains neutral with regard to jurisdictional claims in published maps and institutional affiliations. 